\title{
The evolving role of collaboration in biotechnology
}

\author{
A rapidly changing environment for drug discovery suggests that the \\ collaboration model is just about old enough to become "new."
}

\author{
James M. Gower
}

For executives associated with biotechnology during the past two decades, the remarkable scientific progress made in this industry has in no way been matched by progress in developing business models appropriate to the industry. Despite a neverending series of acronyms to differentiate how this or that business is different, the old adage, the more things change the more they stay the same rings true for biotechnology's management. The practical reason for this is that renaming what you are doing-even if it has changed very little-satisfies the preference $d u$ jour of the usual sources of funding for startup biotechnology firms-venture capital and Wall Street.

This is a very good reason to commit to this exercise, as it is obviously important to raise the necessary cash for building a technology base. But the radical change in the environment for developing drugs demands that early-stage companies use strategies that match their needs rather than trying to satisfy external notions of being categorized as either a service company, a fully integrated pharmaceutical company, or a technology-tool company.

In this regard, returning to the early days of biotechnology and examining what worked and why, can give today's bioentrepreneurs insights into what may prove to be tomorrow's "new" strategy.

\section{Genentech's embarrassment of riches}

Genentech (South San Francisco, CA), in the 1980 s, was more often noted for its competitive stance toward other biotechnology companies than for its cooperative spirit. This reputation was appropriate, but not the whole story. Although not well known, the company generated some of its greatest value through a systematic effort to collaborate with academics and commercial entities.

During its early days, Genentech was so

James M. Gower is president and CEO of Rigel, Inc., 772 Lucerne Drive, Sunnyvale, CA 94086 (jmgower@rigelinc.com). effective at building its research efforts that it faced an embarrassing problem. It was cloning novel proteins at a pace so rapid that it was impossible to thoroughly study the biology of all these discoveries-much less pursue them all as potential development projects. The first approach the company considered using to deal with this problem was guessing. They would line up all the cytokines they had cloned, decide which one was likely to be the superior prospect, and sell off many of the rest. When various banking firms caught wind of this, they appealed to the company to "spin off" a division that would market some of these proteins as novel reagents or development candidates for smaller firms. After considerable debate, the company decided not to pursue this approach.

Instead, the decision was made to collaborate with academic and commercial entities on many of the protein discoveries. In return for a commitment to pursue research on the biological role of these proteins to a point where a decision on drug development could be made, Genentech would grant certain rights to the protein. Occasionally, these turned out to be significant rights, and today there are several products in the clinic as a result of this strategy: $\gamma$-interferon for dermatoses, relaxin, anti-CD-11a, and thrombopoietin.

The point is that Genentech may or may not have gotten to the same point by an alternate strategy, but the benefit to its technological knowledge base, and its profitability, would not have been as great. In hindsight, what started as a simple approach to capture the most value from its excess technological capacity allowed Genentech to leverage this technology and achieve the product pipeline that it enjoys today.

\section{Fast forward}

The riches of today's biotechnology are not cloned proteins but the diverse array of technologies available. In a seminal article on the changes affecting the pharmaceutical industry, Jürgen Drews of Hoffmann-La Roche pointed out that the key issue that needs to be addressed by any company whose goal is drug development is the synthesis of the multiple technologies that represent biotechnology in the $1990 s^{1}$. Drews views this synthesis as having a profoundly positive influence on pharmaceutical drug discovery.

If the integration of genomics, signaling pathway analysis, target validation, combinatorial chemistry, whole-cell functional assays, fluorescence technologies, robotics, miniaturization, and drug screening is the key to obtaining novel drugs from these individual technologies, what are the limiting factors? In the same article, Drews goes on to note that "These opportunities run counter to the economic constraints currently bridling pharmaceutical R\&D plans."

It is frustrating to most biotechnology executives that the growing size and research and development budgets of the newly consolidated pharmaceutical giants are in some ways less accepting of the risk of implementing these new approaches than were the progenitor companies from which they were formed. It is not, however, surprising.

The key pressure on all of the new "mega-pharmas" is the maintenance of an earnings growth that can support their valuations. As this is being written, these valuations are at a historic high-water mark on a profits-to-earnings basis. For all the advantages that can come with greater size, one disadvantage inevitably comes along too: It is harder to grow at the same rate when you are twice as large. Furthermore, the inherent rate of growth depends primarily on the introduction of new products, especially those that take advantage of the technolo- 
gies that allow completely novel mechanisms of action to be exploited.

Few people would argue that greater size produces an increase in corporate speed or innovative ability. The "corporate synergies" that accompany mergers are inevitably in the form of reducing inefficiencies and cost. Paradoxically, these kinds of synergies often result in less speed and innovation in drug discovery. An example of this is that most pharmaceutical companies that have bought genomics databases in the past few years are now struggling to integrate these tools into their drug discovery processes-a task that most are not currently staffed to do and cannot easily afford.

The only possible way out of this drug discovery dilemma is an increasing reliance on outside collaborations. The prevalent attitude in most large pharmaceutical companies is similar to that recently expressed by Daniel Vasella, the president of Novartis (Basel, Switzerland): "External alliances accelerate the pace of drug discovery far more rapidly than a company establishing research capabilities solely in-house."

\section{Integration strategies}

How, then, are we to proceed with this embarrassment of technological riches? Taking a page from Genentech's early history, it is my thesis that small biotechnology companies are better equipped to take on this integration process through collaborations. By sharing their technological riches they will more quickly achieve the integration that the pharmaceutical industry is demanding and build value for the companies involved.

Several technology-based companies are already evolving toward just this type of integrative technology base through acquisition. One example is Millennium (Cambridge, MA), which at an early-stage incorporated biological function assays and pathway elucidation, and later acquired a combinatorial chemistry platform in order to strengthen its genomics base with small molecule discovery. Another example is Axys (South San Francisco, CA), which has rapidly expanded its basic strength in small molecule discovery by adding to it the genomics capacity of Sequana (La Jolla, CA) — also through acquisition.

But given the amount of cash available to biotechnology companies, it is unlikely that acquisition alone will suffice to build totally integrated technology systems. It is much more likely that this will occur through biotech-to-biotech collaborations.

How is this possible? Biotech to biotech arrangements have often been viewed as difficult, if not impossible, because of the inability of either partner to provide cash to the other. This may indeed be an unbridge- able barrier in some circumstances, but certainly not in all.

Many technology-based companies have a far greater breadth to their core technology than they can exploit by themselves. If suitable partners with complementary technology can be found, it is possible to structure arrangements in which both companies can benefit by leveraging each other's technology.

The most beneficial collaborations by far are those in which each company has technology in areas "adjacent" to each other on the drug-discovery continuum. Examples of this adjacency are genomics and signal transduction, and combinatorial chemistry and high-throughput screening. These areas are fairly easy to integrate at a biotechnology level because there is usually significant overlapping expertise in the science base of each company. And it is easier for small companies to assign joint groups to accomplish the project without having to form a committee to organize the collaboration, as often happens in the large pharmaceutical environment. The end result can be a more rapid synthesis of the different technological parts into a drug-discovery process that can provide more valuable product opportunities for both companies.

\section{The Rigel-Neurocrine model}

I have spearheaded just such an approach with Rigel (Sunnyvale, CA). On the one hand, Rigel faced a similar problem to that faced by Genentech over a decade earlier: a broader discovery technology base than it can fully exploit. But on the other hand, as useful as our target validation capability has been, it still stopped short of the full integration that is required for drug discovery. What Rigel needed in the near term was small molecule screening capacity and the chemistry for target optimization.

To address this situation, Rigel entered into a collaboration with Neurocrine Biosciences (San Diego, CA). Neurocrine, for its part, had developed a highly successful, small molecule screening and chemistry effort that resulted in several compounds now in the clinic. The collaboration between Rigel and Neurocrine involves the integration of the genomics, signaling pathway analysis, functional cellular validation, and high-throughput, cell-based florescence screening technology made possible by Rigel's retroviral-based delivery of intracellular combinatorial libraries, with the small molecule screening, robotics, chemical libraries, combinatorial chemistry, and medicinal chemistry of Neurocrine.

Neurocrine gets new target discovery and validation based on genomics and functional pathway mapping in a target area of strategic interest. Rigel gets immediate access to a large chemical library, screening, and the chemistry to add significant value to its technology. Each party is responsible for the funding of its efforts.

Is this approach a grand strategy that ensures success for both companies? No. Is it a new business model begging for an acronym? I hope not. Many companies have accomplished the same objective by alternate means such as mergers. What Rigel and Neurocrine have done, however, is a practical adaptation by both companies to integrate drug discovery technology in the current economic environment. It is also, of course, collaborative. I am sure that both companies would not at all mind seeing the day come when they are competitive in some therapeutic arena with drug products. If that happens, it will mean they have reached another stage in their respective corporate developments. It will also mean that their collaboration has worked.

\section{Conclusions}

Many strategies have been proposed to take advantage of the new approaches available for novel drug discovery. However, as the mega pharmaceutical companies become more and more risk averse, a practical approach is suggested by biotechnology's early history. Collaboration between biotechnology companies recommends itself as an important component of the next stage in biotechnology's evolution. There are three fundamental arguments that suggest this strategy will work: First, no single technology is likely to meet all the drug innovation needs of modern society. Second, no single company or type of company can possibly possess all the answers. Third, the rapid, and sometimes perilous, pace of the changes affecting the economics of biotechnology make the management of these companies more receptive to alternative strategies.

Collaborations of this type should strengthen the industry by helping earlystage companies build their technologies more rapidly. This strategy will also provide partnering platforms for drug-discovery that are more appealing to pharmaceutical partners than any separate technology alone.

In some ways, you could not ask for a better time to be a bioentrepreneur. Good entrepreneurship requires the ability to take advantage of change and use change itself as a strategy. Under these circumstances, the only bad strategies are the ones that ignore the lessons of history and do not consider all the options.

\footnotetext{
1. Drews, J. 1997. Nature Biotechnology
15:1318-1319.

2. Vasella, D. 1997 Nature Biotechnology 15:485-486.
} 